\title{
Tutoria como Estratégia de Aprendizagem nos Cursos de Graduação da UFSM
}

Tutoring as a Learning Strategy in Graduation Courses at UFSM

\author{
Daíse dos Santos Vargas', Marcos Antonio de Azevedo de Campos", \\ Maicon Facco"', Rozieli Bovolini Silveiralv, \\ Yohana Porto Calegari Alvesv, Cleber Bisognin ${ }^{\mathrm{vI}}$
}

\begin{abstract}
RESUMO
O objetivo do presente trabalho é apresentar uma análise realizada sobre o impacto da Central de Tutoria do CCNE no desenvolvimento da aprendizagem dos estudantes da UFSM, especialmente sobre os índices de aprovação. Os dados foram coletados de maio a junho de 2019, sendo realizados nesse período 752 atendimentos em 367 estudantes pertencentes a 47 cursos distintos de 6 Centros de ensino. Verificou-se que em todas as disciplinas de maior procura na Central de Tutoria o número de alunos aprovados foi maior do que os reprovados, com maior destaque para a disciplina de Estatística com índice de aprovação de 88\%. De modo geral, foi observado que os estudantes que buscaram apoio estão, em sua maioria, nas primeiras etapas dos diversos cursos. Esse dado reforça os objetivos da criação da central de tutoria, visto que são nas primeiras semestres dos cursos que o índice de reprovação é alto, contribuindo para a evasão. Em suma, espera-se um aumento na procura dos estudantes por este apoio e, com base nos resultados da análise dos dados apresentada neste trabalho, acredita-se que dar continuidade à Central de Tutoria contribuirá positivamente nos índices de permanência, bem como no êxito dos nossos estudantes.
\end{abstract}

Palavras-chave: Índice de Permanência e Evasão; Aprendizagem entre Estudantes; Ensino.

\begin{abstract}
The aim of this paper is to present the analysis carried out on the impact of CCNE's "Central de Tutoria" on the development of UFSM students' learning, especially on the aprovation indexes. Data were collected from May to June 2019, with 752 attendances during this period in 367 students from 47 different courses at 6 teaching centers. It was found that in all the most sought after subjects in the "Central de Tutoria", the number of students approved was higher than those who failed, with a greater emphasis on the General Statistics discipline with an $88 \%$ pass rate. Overall, it was observed that the students who sought support are mostly in the first stages of the various courses. This data reinforces the objectives of the creation of the "Central de Tutoria", since it is in the early stages of the courses that the failure rate is high, contributing to dropout. In sum, student demand for this support is expected to increase and, based on the results of the data analysis presented in this work, it is believed that continuing the "Central de Tutoria" will positively contribute to the permanence rates as well as the success of our students.
\end{abstract}

Keywords: Escape and Permanence índices; Student Learning; Teaching.

\footnotetext{
' Universidade Federal de Santa Maria, Santa Maria, Brasil. E-mail: dadavargas@hotmail.com.

"Universidade Federal de Santa Maria, Santa Maria, Brasil. E-mail: marcos130694@gmail.com.

III Universidade Federal de Santa Maria, Santa Maria, Brasil. E-mail: maicon_facco@yahoo.com.br.

IV Universidade Federal de Santa Maria, Santa Maria, Brasil. E-mail: rozielisilveira@gmail.com.

v Universidade Federal de Santa Maria, Santa Maria, Brasil. E-mail: yohana.cma@hotmail.com.

v' Universidade Federal de Santa Maria, Santa Maria, Brasil. E-mail: cleber.bisognin@ufsm.br.
} 


\section{INTRODUÇÃO}

O Centro de Ciências Naturais e Exatas (CCNE) é um centro de educação que desenvolve ações de ensino, pesquisa e extensão para 38 cursos de graduação, além de atender estudantes de outros centros da UFSM. São 9 cursos de licenciatura plena, 9 cursos de bacharelado e 1 tecnológico, além dos 19 cursos de pós-graduação. Somente no âmbito presencial de graduação - vinculados ao CCNE - são atendidos 1.992 estudantes com matrícula ativa em 2019 (UFSM, 2019). Porém, o índice de conclusão dos alunos é de apenas $31 \%$ em comparação ao índice de $41 \%$ de conclusão dos alunos totais da UFSM.

Até o ano de 2018, as monitorias no Centro de Ciências Naturais e Exatas (CCNE) eram organizadas e realizadas por monitores selecionados pelos departamentos e somente algumas disciplinas possuíam monitores. Os atendimentos eram realizados em salas isoladas e havia pouca divulgação dos horários dos monitores, favorecendo a baixa procura por atendimentos e os baixos índices de conclusão do curso por parte dos alunos do CCNE.

É preciso considerar que a problemática da evasão é multifatorial, ou seja, a decisão de abandonar o curso geralmente está associada a vários fatores, tais como: ausência de motivação com estudos, vida familiar, contexto escolar ou políticas públicas. Estes fatos revelam que, apesar dos esforços do governo, professores e educadores, os índices de evasão e reprovação são altos. Além disso, muitos estudantes possuem dificuldades sobre determinados conteúdos e na própria organização do estudo, implicando na pouca motivação para o envolvimento nas atividades acadêmicas (ROSÁRIO et al., 2006).Embora as instituições não possam agir sobre todos esses fatores, é necessário repensar e articular ações de permanência a nível institucional. Os cursos das áreas de exatas e naturais, em específico, são desafios complexos que necessitam uma amplitude de estratégias a fim de minimizar os índices da evasão e da reprovação. Neste contexto, para reverter a realidade que envolve a permanência e as características dos cursos ofertados pelo CCNE da UFSM, criou-se o projeto de ensino da Central de Tutoria. 
O projeto "Central de Tutoria no CCNE: espaço de integração e aprendizagem colaborativa" se enquadra numa estratégia metodológica de ensino centrada na participação efetiva dos estudantes na construção do processo de aprendizagem, que se caracteriza por um espaço de ensino personalizado, compartilhado e por tutoria. A aprendizagem requer espaços de prática frequentes e de ambientes ricos em oportunidades. Aprendemos pela mediação de alguém mais experiente e isso acontece quando nos envolvemos de forma mais direta, por questionamento e experimentação. Para isso, o ambiente precisa ser aberto e acolhedor, ajudar os alunos a irem além de onde conseguiriam ir sozinhos, a fim de que cada estudante avance mais na aprendizagem. A interconexão entre a aprendizagem pessoal e colaborativa em um movimento contínuo e ritmado, ajuda o aluno a avançar muito além do que seria possível sozinho (BACICH E MORAN, 2018). A tutoria é entendida como uma estratégia para potencializar a aprendizagem realizada por meio da parceria entre alunos (FRIZON, 2012).

O projeto da Central de Tutoria foi implantado em abril de 2019, com o objetivo de oferecer um serviço de apoio pedagógico amplo e específico para as disciplinas vinculadas ao CCNE, conduzida por tutores das áreas de matemática, física, química, estatística e mais recentemente foi incluída a tutoria para a área de biologia. $\mathrm{O}$ trabalho com os estudantes tutores é coordenado pela Unidade de Apoio Pedagógico (UAP) do CCNE e por professores orientadores de cada área do conhecimento. Assim, este projeto da central de tutoria ampara-se no reconhecimento do papel da instituição no desenvolvimento de políticas de permanência que deem suporte material, social e pedagógico para os estudantes.

Além disso, é necessário repensar o contexto em que o aluno está inserido, bem como, as questões de ensino/aprendizagem, visando uma educação transformadora. Neste contexto, a tutoria oportuniza melhores condições para os sujeitos aprenderem. A alternativa que agora se intenta é estimular os sujeitos a pensarem e agirem de forma estratégica e intencional, autorregulando sua aprendizagem. A ação de autorregular a aprendizagem envolve a participação ativa, construtiva, participativa e autônoma dos sujeitos (VEIGA SIMÃO, 2004). No contexto de uma aprendizagem ativa, cognitiva, 
construtiva, significativa, mediada e autorregulada, a tutoria assume particular importância no ensino universitário, por ser uma proposta que valoriza o desenvolvimento da autonomia, estimula a troca e a parceria no processo de aprender. O trabalho com a tutoria facilita "o desenvolvimento integral dos estudantes, nas suas dimensões intelectual, afetiva, pessoal e social" (VEIGA SIMÃO et al., 2008). Nessa perspectiva buscamos apresentar uma análise realizada sobre a experiência da Central de Tutoria do CCNE e as implicações no desenvolvimento da aprendizagem dos estudantes da UFSM, especialmente sobre os índices de aprovação. Para isso abordaremos a metodologia adotada na pesquisa, os resultados e discussões e por fim, a conclusão.

\section{MATERIAIS E MÉTODOS}

Os dados foram coletados nos meses de maio a julho de 2019 , utilizando o controle de participação da central de tutoria. Este é constituído de informações acadêmicas dos alunos que procuram a central (matrícula, curso, hora de chegada e hora de saída, área e/ou disciplina com dúvidas) e após, assinado pelo tutor que atendeu o aluno. Após o fim do semestre, a Unidade de Apoio Pedagógico do CCNE, utilizando o Sistema de Informações para o Ensino (SIE), verificou a situação de cada um dos alunos atendidos na Central de Tutoria durante o período de análise. Os dados foram digitados em planilhas eletrônicas utilizando o libre office e analisados por meio da análise descritiva, utilizando o R CORE TEAM (2018).

\section{RESULTADOS E DISCUSSÕES}

No primeiro semestre de 2019, em aproximadamente 2 meses de funcionamento, foram realizados 752 atendimentos, entre alunos de graduação e pósgraduação, oriundos de diversos centros acadêmicos da UFSM, dos quais, além do CCNE, podemos citar o Centro de Ciências Rurais (CCR), Centro de Ciências da Saúde (CCS) e o Centro de Tecnologia (CT), como pode ser observado na Tabela 1. 
Tabela 1 - Número de atendimentos por centro de ensino realizadas na Central de Tutoria no período de 13/05/2019 a 18/07/2019

\begin{tabular}{lc}
\hline Centro & Número de tutorias \\
\hline CCNE & 284 \\
CT & 206 \\
CCR & 115 \\
CCS & 80 \\
CCSH & 66 \\
CEFD & 1 \\
\hline Total & 752 \\
\hline
\end{tabular}

Fonte: Central de Tutoria/ Unidade de Apoio Pedagógico-UAP, CCNE, UFSM. Dados do $1^{\circ}$ semestre de 2019.

O centro de ensino mais atendido pela Central de Tutoria (cujos alunos foram mais frequentes na busca por atendimento) foi o Centro de Ciências Naturais e Exatas (CCNE), seguido do Centro de Tecnologia (CT) e Centro de Ciências Rurais (CCR), como mostra a Tabela 1. A menor procura, dentre os centros de ensino que utilizaram os serviços de acompanhamento e atendimento, foi por parte do Centro de Educação Física e Desportos (CEFD), com apenas 1 atendimento.

Observando a Tabela 2, tem-se que houve maior procura por atendimento na Central de Tutoria por parte dos estudantes do curso de Farmácia, seguido pelos estudantes dos cursos de Engenharia Civil, Engenharia Elétrica, Medicina Veterinária, Biologia e Geografia. Demais cursos também procuraram a Central, e se enquadraram na categoria "Outros" por serem menos frequentes e de cursos diversos.

Tabela 2 - Frequência percentual de procura por cursos da UFSM pela Central de Tutoria no período de 13/05/2019 a 18/07/2019

\begin{tabular}{lc}
\hline Curso & Frequência (\%) dos estudantes \\
\hline Farmácia & $9,04 \%$ \\
Engenharia Civil & $7,45 \%$ \\
Engenharia Elétrica & $6,51 \%$ \\
Medicina Veterinária & $6,52 \%$ \\
Geografia Licenciatura & $5,32 \%$ \\
Biologia & $6,12 \%$ \\
Outros & $60,37 \%$ \\
\hline Total & $100,00 \%$ \\
\hline
\end{tabular}

Fonte: Central de Tutoria/ Unidade de Apoio Pedagógico-UAP, CCNE, UFSM. Dados do $1^{\circ}$ semestre de 2019. 
Figura 1 - Número de atendimentos por dia (útil) na Central de Tutoria no período de 13/05/2019 a 18/07/2019

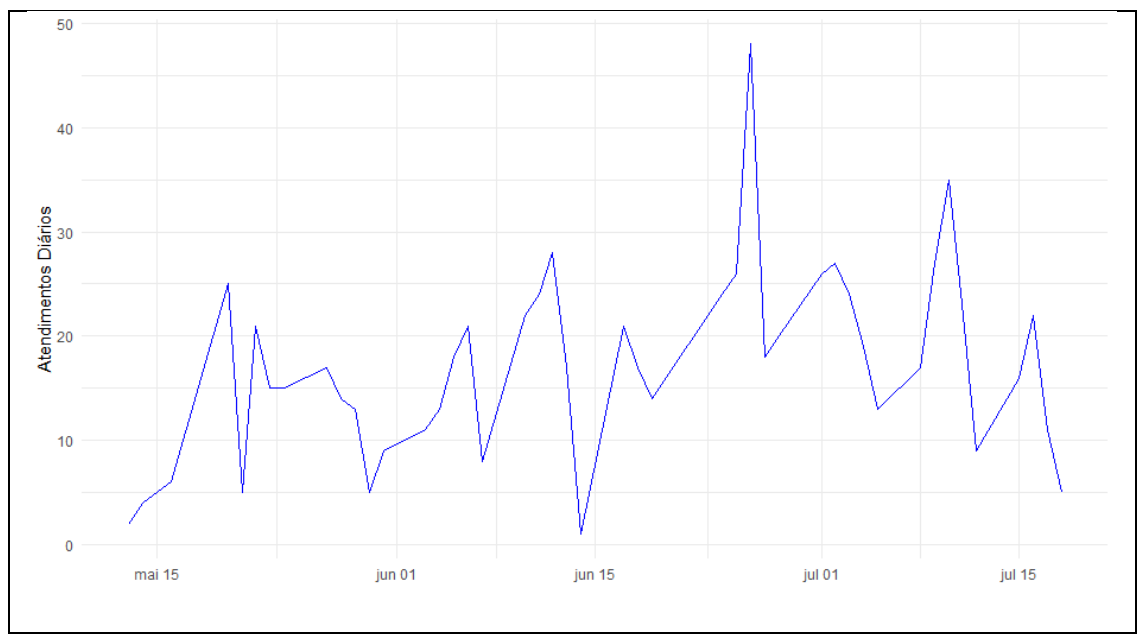

Fonte: Central de Tutoria/ Unidade de Apoio Pedagógico-UAP, CCNE, UFSM. Dados do $1^{\circ}$ semestre de 2019.

Dispondo as tutorias ao longo do tempo é possível observar, por meio da Figura 1, que os números de atendimentos possuem uma leve tendência crescente, ou seja, no decorrer do tempo, quanto mais se aproxima do final do semestre (provas finais e exame), maior é a procura dos alunos na Central de Tutoria, exceto na última semana do semestre (período de 15 a 20 de julho de 2019) quando restam apenas estudantes que necessitam realizar o exame. Também é possível observar uma queda no número de procura da tutoria em todas as sextas-feiras.

Com relação os dias de procura, foi observado que o dia de maior procura no período de maio a julho de 2019 ( $1^{\circ}$ dia do semestre) foi na quarta-feira, seguido de terça-feira e segunda-feira, e o de menor procura na Central de Tutoria foi na sextafeira, conforme apresentado na Tabela 3.

Tabela 3 - Número de atendimentos efetuado aos estudantes da UFSM pela Central de Tutoria no período de 13/05/2019 a 18/07/2019

\begin{tabular}{llllll}
\hline Dias da semana & Segunda & Terça & Quarta & Quinta & Sexta \\
\hline Frequência dos estudantes & 174 & 175 & 203 & 119 & 81 \\
\hline Fonte: Central de Tutoria/ Unidade de Apoio Pedagógico-UAP, CCNE, UFSM. Dados do ${ }^{\circ}$ semestre de 2019.
\end{tabular}

Conforme os dados na Tabela 4, a disciplina que mais teve assessoria solicitada por parte dos estudantes que procuraram a Central de Tutoria foi "Estatística" (ou estatística voltada para a área de formação do acadêmico), com 217 atendimentos (incluindo estudantes que retornaram mais de uma vez). Em segundo lugar, a grande 
procura para sanar dúvidas e melhorar a compreensão do conteúdo foi pela disciplina "Cálculo A", com 180 atendimentos. Em terceiro lugar, houve uma procura relevante também pela disciplina de "Química Geral" (78 atendimentos). Além das já citadas, as disciplinas mais procuradas foram "Álgebra Linear A", "Cálculo B", “Física I", dentre diversas outras.

Tabela 4 - Número de procura por disciplina na Central de Tutoria no período de $13 / 05 / 2019$ a $18 / 07 / 2019$

\begin{tabular}{|c|c|}
\hline Disciplinas & Número de procuras \\
\hline Álgebra Linear A & 41 \\
\hline Cálculo A & 180 \\
\hline Cálculo B & 39 \\
\hline Estatística & 217 \\
\hline Física I & 33 \\
\hline Química Geral & 78 \\
\hline Outras & 164 \\
\hline Total & 752 \\
\hline
\end{tabular}

Além disso, pode ser observado na Tabela 5 que a disciplina com maior tempo de atendimento foi a de Estatística, com um total 371h 07min, sendo as atividades desenvolvidas principalmente as segundas-feiras. Outra disciplina com alto tempo de atendimento foi a de Cálculo A, com um total 237h 58min, sendo desenvolvida nas segundas e terças-feiras a maior parte das suas atividades, seguida por Química Geral, com 98h 20 min, Álgebra Linear, com 43h 11 min, Cálculo B, com 37h 44min e Física I, com $34 \mathrm{~h} 24 \mathrm{~min}$. Para todas as demais disciplinas o total de horas de atendimento foi de $236 \mathrm{~h} 33 \mathrm{~min}$. Ao longo de todo o período foram desenvolvidas um total de $1059 \mathrm{~h}$ $20 \mathrm{~min}$ de atendimento em mais 40 disciplinas.

Tabela 5 - Tempo de atendimento na Central por disciplinas e dias da semana no período de 13/05/2019 a 18/07/2019

\begin{tabular}{|c|c|c|c|c|c|c|c|c|}
\hline \multirow[b]{2}{*}{$\begin{array}{l}\text { Dia da } \\
\text { semana }\end{array}$} & \multicolumn{8}{|c|}{ Disciplina } \\
\hline & Estatística & Cálculo A & $\begin{array}{c}\text { Química } \\
\text { Geral }\end{array}$ & $\begin{array}{c}\text { Álgebra } \\
\text { Linear }\end{array}$ & Cálculo B & Física I & Outros & Total Geral \\
\hline segunda & 107h 08m & $67 \mathrm{~h} 32 \mathrm{~m}$ & $14 \mathrm{~h} 45 \mathrm{~m}$ & 09h $15 \mathrm{~m}$ & 06h 10m & $11 \mathrm{~h} 50 \mathrm{~m}$ & $42 \mathrm{~h} 50 \mathrm{~m}$ & 259h 30min \\
\hline terça & $69 \mathrm{~h} 25 \mathrm{~m}$ & $66 \mathrm{~h} 50 \mathrm{~m}$ & $20 \mathrm{~h} 19 \mathrm{~m}$ & $11 \mathrm{~h} 20 \mathrm{~m}$ & $16 \mathrm{~h} 43 \mathrm{~m}$ & $07 \mathrm{~h} 26 \mathrm{~m}$ & $64 \mathrm{~h} 44 \mathrm{~m}$ & $256 \mathrm{~h} 47 \mathrm{~min}$ \\
\hline quarta & $85 \mathrm{~h} 10 \mathrm{~m}$ & $45 \mathrm{~h} 09 \mathrm{~m}$ & $45 \mathrm{~h} 21 \mathrm{~m}$ & $15 \mathrm{~h} 26 \mathrm{~m}$ & $05 \mathrm{~h} 32 \mathrm{~m}$ & $11 \mathrm{~h} 20 \mathrm{~m}$ & $78 \mathrm{~h} 59 \mathrm{~m}$ & 286h $57 \mathrm{~min}$ \\
\hline quinta & $78 \mathrm{~h} 22 \mathrm{~m}$ & $33 \mathrm{~h} 59 \mathrm{~m}$ & $10 \mathrm{~h} 20 \mathrm{~m}$ & $01 \mathrm{~h} 05 \mathrm{~m}$ & $05 \mathrm{~h} 24 \mathrm{~m}$ & $01 \mathrm{~h} 05 \mathrm{~m}$ & $31 \mathrm{~h} 14 \mathrm{~m}$ & $161 \mathrm{~h} 29 \mathrm{~min}$ \\
\hline sexta & $31 \mathrm{~h} 02 \mathrm{~m}$ & $24 \mathrm{~h} 28 \mathrm{~m}$ & 07h 35m & 06h 05m & 03h 55m & $02 \mathrm{~h} 45 \mathrm{~m}$ & $18 \mathrm{~h} 46 \mathrm{~m}$ & $94 \mathrm{~h} 36 \mathrm{~min}$ \\
\hline Total & $371 \mathrm{~h} \mathrm{07m}$ & $237 \mathrm{~h} \mathrm{58m}$ & $98 \mathrm{~h} 20 \mathrm{~m}$ & $43 \mathrm{~h} 11 \mathrm{~m}$ & $37 \mathrm{~h} 44 \mathrm{~m}$ & $34 \mathrm{~h} 24 \mathrm{~m}$ & 236h 33m & 1059h $20 \mathrm{~m}$ \\
\hline
\end{tabular}

Nos currículos dos diferentes cursos essas disciplinas fazem parte das etapas iniciais do curso, ou seja, os estudantes que buscaram apoio são, em sua maioria, 
estudantes das primeiras etapas do curso. Esse é um dado que reforça os objetivos da criação da central de tutoria, visto que são nas primeiras etapas dos cursos que o índice de reprovação é alto, contribuindo para a evasão. Na tabela 6 são apresentados os dados referentes à situação do acadêmico que participou das atividades na Central de Tutoria. Cabe ressaltar que um acadêmico podia ser atendido na Central de Tutoria em mais de uma disciplina.

Tabela 6 - Situação do acadêmico por disciplina na Central de Tutoria no período de 13/05/2019 a 18/07/2019

\begin{tabular}{lcccc}
\hline \multirow{2}{*}{ Disciplinas } & \multicolumn{4}{c}{ Situação } \\
\cline { 2 - 5 } & Aprovado & Reprovado & Trancamento & Total \\
\hline Álgebra Linear A & 26 & 7 & 0 & 33 \\
Cálculo A & 43 & 28 & 1 & 72 \\
Cálculo B & 13 & 7 & 1 & 21 \\
Estatística & 81 & 10 & 1 & 92 \\
Física I & 13 & 6 & 0 & 19 \\
Química Geral & 33 & 6 & 0 & 39 \\
Outras & 72 & 18 & 1 & 91 \\
\hline Total & 281 & 82 & 4 & 367 \\
\hline
\end{tabular}

Fonte: Central de Tutoria/ Unidade de Apoio Pedagógico-UAP, CCNE, UFSM. Dados do $1^{\circ}$ semestre de 2019.

Como pode ser observado na Tabela 6, em todas as disciplinas com maior procura na Central de Tutoria, o número de alunos aprovados foi maior do que os reprovados. É grande o destaque para a disciplina de Estatística, na qual mais de 88\% dos acadêmicos que foram orientados pelos tutores foram aprovados. Em contrapartida, a disciplina de Cálculo A teve menos de 60\% de índice de aprovação, esse índice está abaixo das demais disciplinas, que no geral obtiveram mais de $76 \%$ de aprovação.

Como a Central de Tutoria é um projeto do CCNE para disciplinas oferecidas em seus departamentos, e também por motivos de localização, o número de atendimento é maior para seus alunos, seguidos pelo CT e o CCR, que são centros que possuem cursos com mais de uma disciplina da área de exatas em seus currículos, principalmente Cálculo e Estatística. Estas são as disciplinas de primeira ou no máximo segundo semestre, ou seja, o aluno apresenta dificuldades pois ainda está se adaptando a universidade e com deficiências vindas do ensino médio. No caso das 
disciplinas de etapas mais avançadas, cujas disciplinas de Cálculo A e Estatística são pré-requisitos, as dificuldades são menores ou mais pontuais.

Há um aumento na procura por atendimento conforme se aproxima do final do semestre, o que pode ser comprovado pela reta de tendência ajusta $Y=0,2374 X+11,762$, onde $Y$ é a variável número de atendimentos e $X$ é a variável dia do atendimento, tendo como $X=1$ o dia 13/02/2019. A procura por atendimento ocorre com maior intensidade nas semanas que antecedem as avaliações finais e exames. Cabe ressaltar que durante este período não houve aumento no número de tutores, mas para que todas as solicitações de atendimento fossem possíveis foram aumentadas as horas em que os tutores estavam à disposição na central de tutoria.

\section{CONCLUSÃO}

Os diversos fatores que envolvem a permanência e as características dos cursos ofertados pelo $\mathrm{CCNE}$, como cursos das áreas de exatas e naturais, são desafios complexos, que necessitam uma amplitude de estratégias a fim de minimizar os índices de evasão e de reprovação. Por isso, a importância das concepções que embasam a implantação da Central de Tutoria, como a personalização, aprendizagem por tutoria e colaborativa, além do desenvolvimento da cultura do estudo ativo.

Uma das ações que favoreceu o desenvolvimento das atividades foi a integração dos tutores, iniciou-se pelo acolhimento, vinculação à Central de Tutoria, apresentação do projeto, a concepção de personalização da aprendizagem, do estudo ativo e o papel esperado enquanto tutores. Como seguimento, os professores orientadores articulam momentos de formação com os tutores, relacionados ao ensino e aprendizagem dos conteúdos específicos das áreas.

Ainda é o momento inicial, porém é possível observar um aumento na procura dos estudantes por este auxílio. A busca pela Central de Tutoria se deu em parte pela auto percepção promovida pelo desenvolvimento do questionário de conhecimentos prévios, realizado após o início do ano letivo. Além disso, foi realizada uma campanha no início do semestre para divulgação e com o objetivo de promover um engajamento acadêmico logo nos primeiros meses, embora seja possível observar que ainda há a 
tendência de procurar apoio nos períodos que antecedem as provas do final do semestre.

O processo de aprendizagem é único e diferente para cada ser humano, cada um aprenderá um conhecimento novo estabelecendo conexões com os conhecimentos que já são conhecidos pelo sujeito. Para que isso ocorra será necessário a aprendizagem seja construída de forma mediada, ou seja, na interação que ocorrerá entre tutor e estudante se estabelecerá uma relação a partir dos conhecimentos científicos a serem aprendidos e aqueles que já sabidos pelo sujeito (VYGOTSKY, 2007). Essa relação de aprendizagem promove o desenvolvimento tanto do estudante que busca apoio quanto do tutor, visto que ambos estão em contínuo desenvolvimento. Além do aspecto cognitivo, da aprendizagem dos conhecimentos científicos, a Central de Tutoria promove o estabelecimento e fortalecimento dos laços afetivos entre os estudantes, refletindo em permanência, visto que um fator de risco para evasão é o desengajamento acadêmico e social progressivo do estudante (RUMBERGER, 2011).

Compartilhamos da concepção de que um dos papéis da instituição é dar ao aluno diferentes caminhos e oportunidades para que construa seu conhecimento e é nesse sentido que o trabalho da Central de Tutoria se ampara. Ou seja, além do espaço da sala de aula, o estudante tem a oportunidade de ensinar e aprender com seus pares, estabelecendo relações de pertencimento aos cursos, ao CCNE e também à UFSM. Nesse sentido é possível observar um aumento na procura dos estudantes por este apoio e acreditamos que ao implementar o acompanhamento em tutoria contribuiremos com os índices de permanência, bem como de êxito dos nossos estudantes.

A organização do espaço da Central de Tutoria permite o desenvolvimento de uma postura ativa frente ao conhecimento, permitindo que o ensino também seja desenvolvido a partir de metodologias ativas. Ou seja, para que seja possível a implementação de metodologias ativas em sala de aula é necessária uma mudança de perspectiva sobre o ensinar e aprender, tornando os sujeitos mais ativos, engajados, motivados para aprender novos conhecimentos que são significativos para si e para a 
comunidade. Dessa forma, este serviço disponibiliza ao discente mais uma oportunidade de construir o conhecimento de forma personalizada, compartilhada e com o apoio de um colega mais experiente. Embora as intervenções realizadas no acompanhamento tutorial não sejam a garantia de sucesso imediato sobre o aluno, a experiência da Central aponta que o maior engajamento do estudante, nas etapas iniciais do semestre, bem como a continuidade favoreceu na aprovação. A maior aprovação nas disciplinas, a aprendizagem de novos conhecimentos e o estabelecimento de vínculos afetivos contribuem para que o estudante permaneça e conclua com êxito seus estudos. Dessa forma pretendemos continuar com os acompanhamentos e estudos dos dados da Central de Tutoria, com o intuito de avaliar a evolução dos resultados e identificar possíveis pontos de melhoria. 


\section{REFERÊNCIAS}

BACICH L, MORAN J (Orgs.). Metodologias ativas para uma educação inovadora: uma abordagem teórico-prática. Porto Alegre: Penso, 2018.

FRISON LBM. Tutoria entre estudantes: uma proposta de trabalho que prioriza a aprendizagem. Revista Portuguesa de Educação. 2012; 25(2): 217-240.

R CORE TEAM [Internet]. R: A language and environment for statistical computing. Vienna, Austria: $\mathbf{R}$ Foundation for Statistical Computing. Disponível em: https://www.R-project.org/.

ROSÁRIO P, NÚÑEZ JC, GONZÁLEZ-PIENDA J. Comprometer-se com o Estudar na Universidade: Cartas do Gervásio ao Seu Umbigo. Campinas: Almerinda S.A, 2006.

RUMBERGER RW. Dropping out: Why students drop out of high school and what can be done about it. Cambridge: Havard University Press, 2011.

UNIVERSIDADE FEDERAL DE SANTA MARIA. Ministério da Educação. UFSM em Números. Disponível em: https://portal.ufsm.br/ufsm-emnumeros/publico/index.html. 2019.

VEIGA SIMÃO AM. O conhecimento estratégico e a auto-regulação da aprendizagem. Implicações em contexto escolar. In: da Silva AL, Duarte AM, Sá I, Veiga Simão AM, Aprendizagem auto-regulada pelo estudante: Perspectivas psicológicas e educacionais. Porto: Porto Editora; 2004. p. 77-87.

VEIGA SIMÃO AM, FLORES MA., FERNANDES S, FIGUEIR C. Tutoria no Ensino Superior: Concepções e práticas. Sísifo - Revista de Ciências da Educação. 2008; 7:75-88.

VYGOTSKY LS. A formação social da mente: o desenvolvimento dos processos psicológicos superiores. $7^{\text {a }}$ ed. São Paulo: Martins Fontes; 2007. 